\title{
Enzymatic Ligation of Antibody and Cell-Penetrating Peptide for Efficient and Cell-Specific siRNA Delivery
}

\author{
Yu Ando \\ Tohoku University \\ Hikaru Nakazawa ( $\sim$ hikaru@tohoku.ac.jp ) \\ Tohoku University \\ Daisuke Miura \\ Tohoku University \\ Mitsuo Umetsu \\ Tohoku University
}

\section{Research Article}

Keywords: Nanobody, siRNA, protein engineering, delivery, antibody, transglutaminase

Posted Date: June 11th, 2021

DOI: https://doi.org/10.21203/rs.3.rs-567665/v1

License: (9) This work is licensed under a Creative Commons Attribution 4.0 International License. Read Full License 


\section{Abstract}

A fusion protein comprising an antibody and a cell-penetrating peptide is a candidate molecule capable of efficient and cell-specific delivery of siRNA into cells in order to reduce the side effects of nucleic acid drugs. However, their expression in bacterial hosts, required for their development, often fails, impeding research progress. In this study, we separately prepared anti-EGFR nanobodies with the K-tag sequence MRHKGS at the C-terminus and arginine-9 (R9) with the Q-tag sequence LLQGS at the N-terminus, and enzymatically ligated them in vitro by microbial transglutaminase to generate Nanobody-R9, which is not expressed as a fused protein in E. coli. Nanobody-R9 exhibited a maximum reaction efficiency of $85.1 \%$, without changing the properties of the nanobody or R9. Nanobody-R9 successfully delivered siRNA into the cells, and the cellular influx of siRNA increased with increase in the ratio of Nanobody-R9 to siRNA. We further demonstrated that the Nanobody-R9-siRNA complex, at a 30:1 ratio, induced RNAi of target mRNA with approximately $52 \%$ efficiency compared to lipofectamine.

\section{Introduction}

RNA interference (RNAi) is a cellular mechanism for post-transcriptional gene regulation mediated by small interfering RNA (siRNA). ${ }^{1,2}$ Specific gene silencing by siRNA holds significant promise for providing new treatment strategies in a wide range of diseases, including cancer and viral infections. ${ }^{3}$ Thus, siRNAbased technology is attractive owing to its

target gene specificity, relatively low siRNA immunogenicity, and simple design. ${ }^{4}$ However, it has two limitations: (1) As siRNA has no cell-type specificity, its medical application would require high doses resulting in high cost and may cause side effects ${ }^{4,5}$ and (2) Because of their hydrophilicity, negative charge, and large molecular weight, siRNA molecules cannot readily cross the cell membrane. ${ }^{6,7}$, are subject to rapid renal clearance and degradation by endogenous RNases, and can be recognized by the innate immune system. ${ }^{8-10}$

To overcome these limitations, recent studies have focused on the combination of a target-specific antibody and a cell-penetrating peptide with siRNA. ${ }^{11-16}$ Antibody is an immune system-related biomolecule that can bind a specific region on an antigen. ${ }^{17}$ As several kinds of antibody molecules have been developed by genetic engineering for disease treatment, ${ }^{17,18}$ it can solve the problem of siRNA specificity. Compared to the IgG type antibodies, small antibodies, such as VHH, scFv, and antibody-like molecules are expected to have stronger penetrating capability and lower immunogenicity ${ }^{19}$ due to the lack of both the constant region and Fc domain. Cell-penetrating peptides typically contain 5-30 amino acids and are mostly positively charged at physiological $\mathrm{pH}$ owing to the presence of several arginine and/or lysine residues. ${ }^{20}$ These peptides can bind to nucleic acids, such as siRNA, through electrostatic interactions. The arginine-9 (R9) motif, a typical cell-penetrating peptide, is more efficient for cellular uptake than other oligomers containing fewer arginine residues or other cationic amino acids such as histidine, lysine, or ornithine.$^{21}$ By combining the technologies of cell-penetrating peptides and antibodies, 
successful cell-specific delivery of siRNA has been reported. ${ }^{15}$ Moreover, no side effects were reported. However, although several cell-penetrating peptide-fused antibodies are functionally expressed as a soluble fraction, ${ }^{15,16}$ in many cases their functional expression was found to be very low in the bacterial host, and were functionalized by unfolding the inclusion bodies ${ }^{11-13}$ or by bio-conjugating. ${ }^{14}$ To expand this technology, it is necessary to develop a method for stable preparation of cell-penetrating peptidefused antibodies.

An effective strategy for functional expression is to separate and express each functional domain and then fuse them in vitro. ${ }^{22}$ This can effectively eliminate the complications related to structure and function, reduce undesirable non-expression, and expression as an insoluble fraction. Reconstruction of each functional unit is generally carried out by bioconjugation using a polymer or particle as support; however, the production of well-defined antibody conjugates is extremely challenging owing to the required selectivity, specificity, and reactivity under physiological conditions. ${ }^{23}$

Recently, a peptide ligation method using sortase and transglutaminase has attracted attention as a method for specifically ligating proteins under mild conditions. ${ }^{24}$ Among them, microbial transglutaminase (MTG; EC 2.3.2.13), belonging to the class transferases, is the most widely used because of its high activity, $\mathrm{Ca}^{+}$independence, and low reverse reaction ${ }^{25}$ The enzyme catalyzes the formation of an isopeptide bond between the $y$-carboxamide group of glutamine residues (donor) and the first-order $\varepsilon$-amine group of other compounds, such as proteins (acceptors of acyl residue). This enzyme can achieve specific ligation by adding peptides, such as K-tag sequence MRHKGS and Q-tag sequence LLQGS, to the protein. ${ }^{26,27}$

In this study, Nanobody-R9, which is not expressed as a fusion protein, was subjected to separate domain expression that were enzymatically ligated by MTG in vitro. Finally, the RNAi of a model siRNA created using the resulting Nanobody-R9MTG ${ }^{\mathrm{MT}}$ was evaluated.

\section{Results}

\section{Expression of Nanobody-R9}

We selected an anti-epidermal growth factor receptor (EGFR) nanobody, ${ }^{28}$ which is a low molecular weight $\mathrm{VHH}$ antibody fragment that can bind to the EGFR on cancer cell surface as a targeting molecule that enables cell-specific delivery of siRNA and the cationic peptide Arginine 9 (R9) as a molecule that can interact with siRNA and penetrate the cell membrane. Using these, a gene coding anti-EGFR nanobody fused with R9 peptide at the C-terminus (Nanobody-R9) was constructed and genetically expressed in E. coli (Figure 1a). Expression of the Nanobody-R9 gene was examined in the culture supernatant fraction, intracellular soluble fraction, and intracellular insoluble fraction post induction under conditions of different temperatures and isopropyl $\beta$-D-thiogalactopyranoside (IPTG) concentrations. Unfortunately, Nanobody-R9 was not expressed in any fraction (Figure 2a, Figure S1). 


\section{Enzymatic synthesis of Nanobody-R9MTG using MTG}

The Nanobody-R9 fusion protein was not expressed in E. coli. Therefore, we synthesized each nanobody and R9 separately and ligated them using MTG. Nanobody with K-tag, which is an MTG recognition sequence at the $\mathrm{C}$-terminus (Nanobody-K, Figure $1 \mathrm{~b}$ ) was expressed in $E$. coli as the intracellular soluble fraction (Figure $2 b$ ). The Nanobody-K-expressing transformant was cultured under the optimal conditions for $16 \mathrm{~h}$ at $28^{\circ} \mathrm{C}$ after IPTG induction, and Nanobody-K was purified using an immobilized metal-ion affinity chromatography (IMAC) and size-exclusion chromatography (SEC). The yield of purified Nanobody-K was calculated to be $27.0 \mathrm{mg} \mathrm{L}^{-1}$-broth. The purified Nanobody-K was ligated with an organically synthesized R9 fused Q-tag (Q-R9-HA), another MTG recognition tag, by MTG. Finally, $5 \mathrm{mM}$ Nanobody-K was mixed with 1-10 times its molar amount (final 5-50 mM) of Q-R9-HA peptide using $50 \mathrm{nM}$ constant of MTG purified by IMAC and SEC (15 $\mathrm{U} \mathrm{mg}^{-1}$ protein). The mixture was incubated at $20^{\circ} \mathrm{C}$ for $6 \mathrm{~h}$. Individually, MTG, Q-R9-HA, and Nanobody-K did not show any product bands in SDS-PAGE analysis (Figure 3a); however, their mixture showed a product band with a molecular weight of $18 \times 10^{-3}$. This molecular weight was similar to that of Nanobody-R9MTG $\left(19.1 \times 10^{-3}\right)$, suggesting that Nanobody-R9MTG was successfully prepared. Western blot analysis also showed that Nanobody-R9MTG could be detected by HA-tag and His-tag (Figure $3 \mathrm{~b}$ and $\mathrm{c}$ ), indicating that the product was a complex comprising Nanobody-K and Q-R9-HA. Notably, unreacted Q-R9-HA was not detected by HA-tag detection. Presumably, Q-R9-HA passes through the nitrocellulose membrane due to its small size during the transfer. The band size of Nanobody-R9MTG increased slightly and that of unreacted Nanobody-K decreased in the reaction mixture with increasing concentration of Q-R9-HA. When the molar ratio of Nanobody:R9 was 1:1, 1:3,1:5, 1:7, and 1:10, reaction efficiency of the nanobody was calculated to be $30.4,62.7,77.7,83.0$, and $85.1 \%$, respectively, based on the signal intensity of band in SDS-PAGE, respectively.

To prove that MTG does not react with lysine residues in the nanobody sequence, we created a nanobody in which the $\mathrm{K}$ tag was replaced by a lysine-free Cmyc' tag. No ligated product was found (Figure S2), indicating that MTG does not react with the nanobody and specifically reacts with the K-tag. The unreacted peptide and Nanobody-K in the solution after Nanobody-R9MTG synthesis were removed by IMAC purification, and the purified Nanobody-R $9^{\mathrm{MTG}}$ was used in the subsequent experiments.

\section{Secondary structure analysis of Nanobody-R9MTG using circular dichroism spectroscopy}

To confirm whether the Nanobody-R9MTG retained its structure compared to the parent Nanobody-K, both structures were analyzed by circular dichroism spectrometry. As shown in Figure 4a, the Nanobody-R9MTG structure showed a typical b sheet-rich immunoglobulin fold, and no significant change was observed when compared with the structure of Nanobody-K. This result indicates that the nanobody part of Nanobody-R9 ${ }^{M T G}$ formed a correct structure.

\section{Binding affinity of Nanobody-R9MTG to EGFR}


The binding affinity of Nanobody-R9MTG to EGFR was evaluated to demonstrate that the crosslinking of R9 to nanobody did not affect the function of the nanobody. Nanobody-K and Nanobody-R9MTG, modified with FITC, were mixed with EGFR-positive A431 cells, and the fluorescent intensity of the interacting cells was analyzed by flow cytometry. Both nanobodies were strongly bound to A431 cells. The fluorescence intensity of Nanobody-R9MTG showed only a slight decrease compared to that of Nanobody-K, indicating that there was no significant change in the binding affinity of the nanobody to EGFR after R9 crosslinking (Figure $4 \mathrm{~b}$ ). We demonstrated that the nanobody module of Nanobody-R9MTG is available to target EGFRpositive cells. The $K \mathrm{~d}$ of Nanobody-R MTG $^{\mathrm{MT}}$ was calculated as $63 \pm 13 \mathrm{nM}$ by flow cytometry analysis under various concentrations of the protein (Figure 4c).

\section{Interaction of Nanobody-R9MTG with siRNA}

The interaction between Nanobody-R9MTG and siRNA was evaluated using an Electrophoretic mobility shift assay. Briefly, 5 pmol Cy5-modified siRNA (Cy5-siRNA) and 10-150 pmol (2-30 times equivalent) Nanobody-R9MTG were mixed at $20-25^{\circ} \mathrm{C}$ for $30 \mathrm{~min}$, and the reaction mixture was analyzed by agarose gel electrophoresis (Figure 5). When Nanobody-R9MTG concentration was at least 6-fold higher than that of siRNA, the mobility of Cy5-siRNA decreased. This result showed that Cy5-siRNA interacted with Nanobody-R9MTG. Furthermore, the mobility of Cy5-siRNA decreased gradually with increasing ratio of Nanobody-R9MTG to Cy5-siRNA, indicating that multiple Nanobody-R9MTG molecules can bind to one siRNA. In addition, unreacted siRNA was not detected when using 6-30 times Nanobody-R9MTG , indicating that all siRNA interacted completely with Nanobody-R9MTG. Thus, we demonstrated the availability of the R9 peptide in Nanobody-R9MTG to capture siRNA.

\section{siRNA delivery into EGFR-positive cells with Nanobody-R9MTG}

We demonstrated the that siRNA could be successfully delivered into EGFR-positive cells using Nanobody-R9MTG using confocal microscopy (Figure 6). After incubating the final $20 \mathrm{nM}$ Cy5-siRNA and its 6-, 14-, and 30 -fold Nanobody-R9MTG concentration for $30 \mathrm{~min}$ at $20-25^{\circ} \mathrm{C}$, these mixtures were added to the pre-cultured A431 cells and incubated at $37{ }^{\circ} \mathrm{C}$ in $5 \% \mathrm{CO}_{2}$. After incubation for $6 \mathrm{~h}$, cell nuclei and cell membranes were pre-stained with DAPI, and Cell Mask Green Plasma in the cell was observed by confocal microscopy. We used Lipofectamine 2000, a high-efficiency DNA and RNA transfection reagent, as a positive control. We found that when siRNA alone was added to the cell, Cy5 fluorescence was not observed in the cells; whereas when Naobody-R9MTG and siRNA were added to the cell, siRNA was detected in the cells under all conditions. This result showed that the addition of Naobody-R9MTG improved the efficiency of siRNA delivery. In addition, the fluorescence intensity of Cy 5 increased with increasing dose of Nanobody-R9MTG, indicating that the siRNA is more likely to be taken up into cells under conditions of Nanobody-R9MTG multivalency. Under all conditions, the uptake efficiency of the Nanobody-R9MTG_siRNA complex by cells was lower than that of the positive control 
lipofectamine. However, the addition of Nanobody-R9MTG_siRNA to EGFR-negative HEK293 cells under the optimal conditions did not deliver siRNA into the cells (Figure S3).

\section{RNAi with Nanobody-R9MTG_siRNA complex}

To investigate whether the Nanobody-R9 ${ }^{\mathrm{MTG}}$-siRNA complex can suppress the production of the target protein, a Nanobody-R9MTG_siRNA complex targeting glyceraldehyde-3-phosphate dehydrogenase (GAPDH) as a model was prepared and RNAi was evaluated (Figure 7). 20 pmol of siRNA was mixed with 120 or 600 pmol of Nanobody-R9MTG and the complex was added to cultured A431 cells. After $72 \mathrm{~h}$, the amount of GAPDH in the cell extract was detected by western blot analysis with anti-GAPDH antibody (Figure 7a). When siRNA alone was used, the GAPDH production was not changed compared to that of $\beta$-actin, a housekeeping protein. However, when the Nanobody-R9MTG_siRNA complex was used, the production of GAPDH was significantly reduced. This result indicates that the RNAi of the specific gene was significantly more efficient when using the Nanobody-R9MTG_-siRNA complex than that with siRNA alone. GAPDH production was calculated from the band intensity in western blot as a percentage of GAPDH expression compared to $\beta$-actin. As a result, the production levels of GAPDH in 6- and 30-fold samples were $87.8 \%$ and $64.6 \%$, respectively, indicating that they were suppressed by $12.2 \%$ and $35.4 \%$, respectively. This performance was not as good as of that of Lipofectamine (60.4\%). Moreover, we confirmed that the gene expression was suppressed at the transcription level using RT-PCR (Figure 7b). We found that the expression pattern of the transcripts correlated with the signal intensity of western blot analysis. Thus, GAPDH production was affected by RNAi-mediated transcriptional repression.

\section{Discussion}

\section{Construction of fusion protein using transglutaminase}

In recent years, researchers have actively focused on cell-specific oligonucleotide therapeutics using antibodies that have fewer side effects. However, one of the obstacles to developing an antibody-siRNA as cell-specific oligonucleotide therapeutic is the difficulty in functional expression of a fusion protein of antibody and cell-penetrating peptide in E. coli. Therefore, it is necessary to establish a stable antibodycell-penetrating peptide fusion method. Here, we solved this problem by enzymatic fusion using MTG. We focused on the nanobody-fused $\mathrm{R} 9$ peptide that is not expressed in $E$. coli but is expected to induce tissue-specific RNAi as a fusion protein. In this study, antibody and R9 were prepared separately and expressed successfully in E. coli. Although the underlying mechanism is not clear, the fusion of R9 peptide to antibody appears to induce expression in E. coli. In the present study, the K-tag peptide did not prevent expression, at least in the R9 peptide. Similar examples of easy expression due to functional domain division have been previously reported in a wide range of proteins, including enzymes and

antibodies. $^{29,30}$ The domain separating process for $E$. coli expression was applied to generate NanobodyR9. MTG efficiently catalyzed the enzymatic ligation of the antibody and R9. The reaction was completed by simply mixing the two at room temperature with PBS as the solvent. 
Nanobody-R9MTG production increased with increasing molar ratio of Q-R9-HA to Nanobody-K and plateaued at when the ratio of Nanobody-K: Q-R9-HA was 1:7. The reaction efficiency, based on band intensity, under optimum conditions was calculated to be $85.1 \%$ when the ratio of Nanobody-K: Q-R9-HA was 1:10. On the other hand, with increasing dosage of R9 peptide, the amount of unreacted R9 increased. As a result, at the molar ratio of $1: 10,90 \%$ of R9 remained unreacted. However, Nanobody-R9 and residual R9 peptides could be easily removed by gel filtration chromatography. Moreover, the R9 peptide was recovered from the reaction mixture and could be reused (Figure S4). In addition, the structure of Nanobody did not change much before and after peptide ligation; they showed almost the same function as that of the unfused nanobody. In this design, the domains were linked via a $\mathrm{G}_{4} \mathrm{~S}$ linker to avoid steric hindrance. Our design was successful and worked well in this study.

\section{Binding of Nanobody-R9 to siRNA and delivery of siRNA to cells}

Nanobody-R9 interacted with siRNA and the results showed that not only Nanobody, but also R9, was found to work after enzymatic ligation by MTG. Several Nanobody-R9 molecules could bind to a single siRNA molecule. Even though the valence of Nanobody-R9 exceeded 30, it was still increasing. Such a tendency has also been reported in previous studies. ${ }^{14,20,31}$ The cells were incubated Nanobody-R9siRNA containing two different molar ratios $(1: 6,1: 30)$ of siRNA and Nanobody-R9. siRNA alone did not enter the cell, showing its low efficiency of introduction into cells because the negative charges of the cell membrane and the siRNA repel each other. ${ }^{32}$ However, the Nanobody-R9-siRNAs can facilitate the entry of the siRNA into the EGFR positive cells and with increasing valency of Nanobody-R9 to siRNA, the amount of Nanobody-R9-siRNA entering the cells increased. We hypothesized two reasons for this increase in siRNA uptake with increasing valence of Nanobody-R9. One reason for this increased uptake frequency was the increased affinity of nanobodies to EGFR due to the multivalent effect, which facilitates EGFR-dependent endocytosis. A similar result was previously reported that the rate and level of antibody internalization largely depends on the affinity of the engineered antibodies towards FGFR1, as high-affinity antibodies display the fastest internalization kinetics. ${ }^{33}$ The other reason is the cancelling of the negative charge of the membrane by accumulation of R9 around the siRNA. Wang et al. reported that R9 peptide and siRNA bound, through electrostatic interaction, at a charge ratio of about 1:12 (1:56 molar ratio), resulting inefficient delivery of siRNA into cells. The net charge of Nanobody R9 would be biased towards the positive charge beyond cancellation of the negative charge of siRNA. Electrostatic interaction with the membrane of Nanobody-R9-siRNA induces macropinocytosis. ${ }^{34}$ Under optimal conditions, the amount of transferred Nanobody-R9 to the cell was approximately $50 \%$ of that of Lipofectamine 2000. This result is consistent with previous reports. ${ }^{20}$

\section{RNAi with Nanobody-R9MTG}

Using the Nanobody-R9 and GAPDH siRNAs, we investigated RNAi induction; GAPDH production was reduced by adding Nanobody-R9MTG _ siRNA, proving that RNAi could be triggered. We constructed a molecule that facilitated GAPDH siRNA transfer into the nucleus, specifically suppressing GAPDH 
transcription, and finally suppressed 35\% GAPDH production. Lu et al. has previously reported that the addition of scFv-R9/Her2 siRNA suppressed $\sim 45 \%$ of Her2 expression, whereas scFv/Her2 siRNA did not alter Her2 expression. ${ }^{35}$ Examination at the transcriptional level revealed similar results, suggesting that the reduction in transcription was related to a reduction in protein production. Moreover, increasing the valency of R9 on siRNA increased the effect of RNAi. siRNA has to interact with RNA induced silencing complex (RISC) to work in the nucleus or cytoplasm, suggesting that the increased valency of R9 may be involved in endosomal escape. This may enhance the proton sponge effect proposed by Boussif et al. ${ }^{36}$ Boussif et al. described that the amino group of the cationic peptide that had been deprotonated extracellularly $(\mathrm{pH} \mathrm{7.4)}$ is first protonated inside the late endosome $(\mathrm{pH} \sim 5.5)$. Thereafter, siRNA escape would occur due to counterion influx and osmotic pressure increase, resulting in endosomal destabilization. We hypothesize that it to be triggered by increase in amino group of Arg with internalization of a large amount of R9 in the present study.

In conclusion, the fusion protein Nanobody-R9, which is not expressed under normal conditions, was expressed separately as each functional domain and then ligated with MTG to successfully express the fusion protein. The coupling efficiency reached $85 \%$. Our study provides a basis for the generation of such functional fusion proteins that have many potentials but are not expressed.

\section{Methods}

\section{Materials}

siRNAs: Silencer ${ }^{\circledR}$ Select GAPDH Positive Control siRNA was purchased from Thermo Fisher Scientific (San Jose, CA, USA). These siRNAs were used after labeling with Cy 5 using the Labe/ IT ${ }^{\circledR}$ siRNA Tracker ${ }^{\mathrm{TM}}$ Cy ${ }^{\circledR} 5$ Kit (Mirus Bio LLC, Madison, WI, USA). Peptides: The synthetic peptide Q-tag-R9-HA (5'-AcetylLLQGRRRRRRRRRYPYDVPDYA-COOH-3') was purchased from Eurofins Genomics (Ebersberg, Germany). Cell lines: A431 human epidermoid carcinoma cells were obtained from the Cell Resource Center for Biomedical Research, Institute of Development, Aging, and Cancer, Tohoku University (Sendai, Japan). HEK293 cells were obtained from the Japanese Collection of Research Bioresources Cell bank (Osaka, Japan). These cells were cultured in RPMI 1640 medium with L-glutamine and sodium bicarbonate (liquid, sterile-filtered, suitable for cell culture; Sigma Aldrich, St. Louis, MO, USA) supplemented with 10\% fetal bovine serum (Biowest, France) and 1× Anti-Anti (Gibco, Thermo Fisher Scientific, Waltham, MA, USA).

\section{Construction of expression vector}

The gene encoding anti-EGFR nanobody from la1 llama antibody was amplified to fuse the K-tag-His-tag (GGGGSMRHKGSHHHHHH), R9-His-tag, (GGGGSRRRRRRRRRHHHHHH), or Cmyc'-His-tag (TMFLISEEDLQHHHHHH) to its C-terminus by polymerase chain reaction (PCR). PCR products and pRA expression vector were digested with $\mathrm{Ncol}$ and Spel in case of K-tag-His-tag and R9-His-tag, or with Ncol 
and Sacll in case of Cmyc'-tag, and ligated to each other at $16^{\circ} \mathrm{C}$ for 30 min to generate pRA-Nb-K-His, pRA-Nb-R9-His, and pRA-Nb-Cmyc'-His expression vectors.

The gene encoding MTG from Streptomyces mobaraensis (accession number DQ132977) fused 6× Histag to the C-terminus synthesized by Thermo Fisher Scientific and was amplified by PCR to generate Ncol and EcoRI sites. PCR products and the pET22b expression vector were digested with $\mathrm{Ncol}$ and EcoRI, followed by ligation at $16{ }^{\circ} \mathrm{C}$ for 30 min to generate the pET22b (+)-MTG-His expression vector.

\section{Expression and purification of recombinant antibodies}

E. coli BL21 (DE3) transformants harboring pRA-Nb-K-His, pRA-Nb-R9-His, and pRA-Nb-Cmyc'-His were cultured at $28{ }^{\circ} \mathrm{C}$ in flasks containing $2 \times$ YT medium supplemented with $100 \mathrm{mg} \mathrm{mL}^{-1}$ ampicillin, and protein expression was induced by adding $1 \mathrm{mM}$ IPTG when the absorbance of the culture at $600 \mathrm{~nm}$ reached 0.8 . After incubation at $28^{\circ} \mathrm{C}$ for $16 \mathrm{~h}$, the culture supernatant and intracellular soluble fractions and insoluble fractions were collected and evaluated by SDS-PAGE and western blot. Proteins were purified using IMAC (Ni Sepharose 6 Fast Flow; GE Healthcare Bio-Sciences AB, Uppsala, Sweden), and SEC (HiLoad 26/600 Superdex 75 prep grade; GE Healthcare Bio-Sciences AB).

\section{Expression and Purification of MTG}

Transformed E. coli BL21 (DE3) cells harboring expression plasmids encoding MTG were incubated in $2 \times$ YT medium containing $100 \mathrm{~g} \mathrm{~mL}^{-1}$ ampicillin at $37^{\circ} \mathrm{C}$, and expression of recombinant antibodies under the control of the T7 promoter was induced by adding $0.4 \mathrm{mM} \mathrm{IPTG}$ when the absorbance of the culture at $600 \mathrm{~nm}$ was 0.8 . After additional incubation at $20^{\circ} \mathrm{C}$ for $30 \mathrm{~h}$, the bacterial supernatant was collected and purified using IMAC and SEC (HiLoad 26/600 Superdex 200 prep grade; GE Healthcare BioSciences $A B)$.

\section{MTG-mediated fusion of Nanobody-K and Q-R9 and purification of Nanobody-R9MTG}

The fusion of each nanobody and R9 peptide was conducted by mixing $5 \mu \mathrm{M}$ Nanobody-K (or NanobodyCmyc'), 5-35 $\mathrm{MM}$ Q-R9, and $0.03 \mathrm{U} / \mathrm{mL}$ MTG in PBS (pH 7.4) at $20^{\circ} \mathrm{C}$ for $6 \mathrm{~h}$. The reaction product was analyzed by SDS-PAGE and western blot analysis, and reaction efficiency was evaluated based on band intensity using Image Quant Las 4000 (GE Healthcare Bio-Sciences AB). The reaction product was purified using IMAC ( $1 \mathrm{~mL}$ His trap HP; GE Healthcare Bio-Sciences AB) with 0-400 mM imidazole gradient in $1 \times$ PBS containing $750 \mathrm{mM} \mathrm{NaCl}$ to remove MTG and unreacted Q-R9 and Nanobody-K.

\section{Electrophoretic mobility shift assay}

Nanobody-R9MTG and 5 pmol Cy5-siRNA were mixed, with increasing amount of Nanobody-R9 in PBS. The mixture was incubated at $4{ }^{\circ} \mathrm{C}$ for $30 \mathrm{~min}$ and then electrophoresed on $2 \%(\mathrm{w} / \mathrm{v})$ agarose gels in TAE buffer. The mobility shift of the siRNA band was visualized on Cy 5 detected using a UV-transilluminator. 
The specific binding of Nanobody-R9MTG or Nanobody-K to EGFR on the cell surface was analyzed by flow cytometry. For this, forty-seven $\mathrm{nM}$ of Nanobody-R ${ }^{\mathrm{MTG}}$ or Nanobody-K were labeled with fluorescein Labeling kit- $\mathrm{NH}_{2}$ (Dojindo, Inc. Kumamoto) and mixed with EGFR-positive $2 \times 10^{6} \mathrm{~A} 431$ cells. The mixtures were incubated for 60 min on ice. The cells were washed three times with PBS containing $0.01 \%$ bovine serum albumin and analyzed by flow cytometry (FACS Accuri 6; BD Biosciences, Franklin Lakes, NJ, USA).

\section{Confocal microscopy}

A431 cells $\left(1.5 \times 10^{5}\right)$ were grown on glass bottom dishes (Matsunami Glass Ind., Ltd., Japan) in RPMI medium for $24 \mathrm{~h}$ at $37^{\circ} \mathrm{C}$ and $5 \% \mathrm{CO}_{2}$. Then, 50 pmol Cy 5 -siRNA was mixed with 300 or 1,500 pmol Nanobody-R9MTG in PBS (final volume $500 \mu \mathrm{L}$ ), and the mixture was incubated at $4{ }^{\circ} \mathrm{C}$ for 60 min. Lipofectamine2000 was used as a positive control. The reaction mixture was added to the culture medium of A431 cells followed by incubation at $37^{\circ} \mathrm{C}$ for $6 \mathrm{~h}$ in the cell incubator. The cells were washed twice with PBS and stained with $1 \mathrm{mg} \mathrm{mL}^{-1}$ 4',6-diamidino-2-phenylindole (DAPI; Sigma-Aldrich) and Cell Mask $^{\mathrm{TM}}$ Green plasma Membrane stain (FITC detection, Thermo Fisher Scientifics). After staining, the cells were observed under a confocal microscope (FV1200-D; Olympus, Japan).

\section{Western blot}

A431 cells $\left(1.5 \times 10^{4}\right)$ were grown on 24 -well plates in RPMI medium for $24 \mathrm{~h}$ at $37^{\circ} \mathrm{C}$. Then, 20 pmol Cy5siRNA was mixed with 120 or 6000 pmol Nanobody-R9MTG in PBS (final volume $150 \mu \mathrm{L}$ ) and incubated at $4{ }^{\circ} \mathrm{C}$ for $60 \mathrm{~min}$. Lipofectamine 2000 was used as a positive control. The mixture was added to the culture medium of A431 cells and incubated at $37^{\circ} \mathrm{C}$ for $72 \mathrm{~h}$ in a cell incubator. Thereafter, the cells were washed in PBS and lysed directly with RIPA buffer (Nacalai Tesque, Kyoto, Japan). The extracted proteins were then transferred onto nitrocellulose membranes (Millipore, Bedford, MA, USA), which were incubated with primary antibodies against GAPDH (1:7,500; MBL, Nagoya, Japan) and $\beta$-actin (1:7,500; MBL), followed by incubation with horseradish peroxidase (HRP)-conjugated goat anti-mouse Ig secondary antibody (1:7,500; Proteintech group, Inc., Tokyo, Japan). Finally, Chemi-Lumi One (Nacalai Tesque) was used to visualize the protein bands.

\section{Real time PCR}

First, 120 or 6000 pmol Nanobody-R9MTG was mixed with 20 pmol siRNA in $150 \mu \mathrm{L}$ of PBS and incubated at $4{ }^{\circ} \mathrm{C}$ for $60 \mathrm{~min}$. A431 cells $\left(3.0 \times 10^{4}\right)$ were grown on 24-well plates (Costar 24 well 3524, CORNING, NY, USA) in RPMI medium at $37^{\circ} \mathrm{C}$ overnight. The cells were then washed in PBS and incubated with 250 $\mathrm{mL}$ Nanobody-R9 siRNA (with final concentration of $40 \mathrm{nM}$ siRNA) in $250 \mu \mathrm{L}$ RPMI medium at $37^{\circ} \mathrm{C}$ for $24 \mathrm{~h}$. Thereafter, cells were washed three times in PBS. Next, the cells were lysed using SingleShotCell Lysis kit (Bio-Rad laboratories, inc. CA, USA) and cDNA was synthesized using Advanced cDNA synthesis kit for RT-PCR (Bio-Rad laboratories, inc.) according to the manufacturer's instructions. RT-PCR of GAPDH transcripts was performed using a mixture of $1 \mu \mathrm{L} c D N A, 5 \mu \mathrm{L} 5 \times$ iScript SYBR Green supermix (Bio-Rad 
laboratories, inc.), 10 pmol $\mu \mathrm{L}^{-1}$ GAPDH Fw primer: 5'-GTCTCCTCTGACTTCAACAGCG-3' and GAPDH Rv primer: 5'-ACCACCCTGTTGCTGTAGCCAA-3' and nuclease-free water by thermal cycling under following conditions: 40 cycles of $95^{\circ} \mathrm{C}$ for $30 \mathrm{~s}, 95^{\circ} \mathrm{C}$ for $10 \mathrm{~s}$, and $60^{\circ} \mathrm{C}$ for $30 \mathrm{~s}$. The cDNA amount was calculated using the $\triangle \triangle \mathrm{Ct}$ method (ACTIN Fw primer: 5 '-CACCATTGGCAATGAGCGGTTC-3' and ACTIN Rv primer: 5'-AGGTCTTTGCGGATGTCCACGT-3' were used to RT-PCR of ACTIN transcripts as a house keeping).

\section{Abbreviations}

IMAC, immobilized metal ion affinity chromatography; SCFv, single-chain variable fragments; SEC, size exclusion chromatography; EGFR, epidermal growth factor receptor; SDS-PAGE, Sodium dodecyl sulfate polyacrylamide gel electrophoresis; MTG, microbial transglutaminase

\section{Declarations}

\section{Acknowledgement}

This work was partly supported by Scientific Research Grants from the Ministry of Education, Culture, Sports, Science and Technology of Japan [grant numbers $18 \mathrm{~K} 04842$ (H.N.); 16H04570, 16K14483, $20 \mathrm{H00315}$ (M.U.), 19 K22628 (T.M.), and 24000011 (I.K.)] and Japan Agency for Medical Research and Development (M.U., JP18am0301016h000).

\section{Author contributions}

M.U., Y.A. and H.N. designed the study.; Y.A. and H.N. wrote the manuscript. D.M. and Y.A. performed the experiment and analyzed the data.

\section{Conflict of Interest}

The authors declare no competing financial interest.

\section{References}

1. Davidson, B.L. \& McCray. P.B.Jr. Current prospects for RNA interference-based therapies. Nat. Rev. Genet. 12, 329-340 (2011).doi: 10.1038/nrg2968

2. Wilson, R.C. \& Doudna J.A. Molecular mechanisms of RNA interference. Annu. Rev. Biophys. 42, 217-239 (2013). doi:10.1146/annurev-biophys-083012-130404

3. Whitehead, K.A., Langer, R. \& Anderson, D.G. Knocking down barriers: advances in siRNA delivery. Nat. Rev. Drug Discov. 8, 129-138 (2009).doi: 10.1038/nrd2742

4. McNamara, J.O.2nd, Andrechek, E.R., Wang, Y., Viles, K.D., Rempel, R.E., Gilboa, E., Sullenger, B.A. \& Giangrande, P.H. Cell type-specific delivery of siRNAs with aptamer-siRNA chimeras. Nat. Biotechnol. 24, 1005-1015 (2006). doi: 10.1038/nbt1223 
5. Castanotto, D. \& Rossi, J.J. The promises and pitfalls of RNA-interference-based therapeutics. Nature 457,426-433 (2009).doi: 10.1038/nature07758

6. Wang, J., Lu, Z., Wientjes, M.G. \& Au, J.L. Delivery of siRNA therapeutics: barriers and carriers. AAPS J. 12, 492-503 (2010).doi: 10.1208/s12248-010-9210-4

7. Dominska, M. \& Dykxhoorn, D.M. Breaking down the barriers: siRNA delivery and endosome escape. J. Cell Sci. 123, 1183-1189 (2010).doi: 10.1242/jcs.066399

8. Das, M., Musetti, S. \& Huang, L. RNA interference-based cancer drugs: the roadblocks, and the "delivery" of the promise. Nucleic Acid Ther. 29, 61-66 (2019).doi: 10.1089/nat.2018.0762

9. Zhou, J. \& Rossi, J.J. Mechanisms and barriers to RNAi delivery. Advanced delivery and therapeutic applications of RNAi (ed. Cheng, K. and Mahato, R.I.) 3-17 Chapter 1, John Wiley and Sons, Ltd. (2013) doi.org/10.1002/9781118610749.ch1

10. Meng, Z. \& Lu, M. RNA interference-induced innate immunity, offtarget effect, or immune adjuvant? Front Immunol. 8, 331 (2017).doi: 10.3389/fimmu.2017.00331

11. Li, X., Stuckert, P., Bosch, I., Marks, J.D. \& Marasco, W.A. Single-chain antibody-mediated gene delivery into ErbB2-positive human breast cancer cells. Cancer Gene Ther. 8, 555-565 (2001). doi:

10.1038/sj.cgt.7700337

12. Song, E., Zhu, P., Lee, S.K., Chowdhury, D., Kussman, S., Dykxhoorn, D.M., Feng, Y., Palliser, D., Weiner, D.B., Shankar, P., Marasco, W.A. \& Lieberman, J. Antibody mediated in vivo delivery of small interfering RNAs via cell-surface receptors. Nat. Biotechnol. 23, 709-717 (2005). doi:

$10.1038 / \mathrm{nbt} 1101$

13. Dou, S., Yao, Y.D., Yang, X.Z., Sun, T.M., Mao, C.Q., Song, E.W. \& Wang, J. Anti-Her2 single-chain antibody mediated DNMTs-siRNA delivery for targeted breast cancer therapy. J. Control Release 161, 875-883 (2012). doi: 10.1016/j.jconrel.2012.05.015.

14. Kumar, P., Ban, H.S., Kim, S.S., Wu, H., Pearson, T., Greiner, D.L. Laouar, A., Yao, J., Haridas, V., Habiro, K., Yang, Y.G., Jeong, J.H., Lee, K.Y., Kim, Y.H., Kim, S.W., Peipp, M., Fey, G.H., Manjunath, N., Shultz, L.D., Lee, S.K. \& Shankar, P. T Cell-Specific siRNA Delivery Suppresses HIV-1 Infection in Humanized Mice. Cell 134, 577-586 (2008). doi: 10.1016/j.cell.2008.06.034

15. Peer, D., Zhu P., Carman, CV., Lieberman, J. \& Shimaoka, M. Selective gene silencing in activated leukocytes by targeting siRNAs to the integrin lymphocyte function-associated antigen-1. Proc. Natl. Acad, Sci. U.S.A. 104, 4095-4100 (2007). doi.org/10.1073/pnas.0608491104

16. Lu, Y., Liu, L., Wang, Y., Li, F., Zhang, J., Ye, M., Zhao, H., Zhang, X., Zhang, M., Zhao, J., Yan, B., Yang, A., Feng, H., Zhang, R. \& Ren, X. siRNA delivered by EGFR-specific scFv sensitizes EGFR-TKI-resistant human lung cancer cells. Biomaterials 76, 196-207 (2016). doi: 10.1016/j.biomaterials.2015.10.036

17. Scott, A.M., Volchok, J.D. \& Old, L.J. Antibody therapy of cancer, Nat. Rev. Cancer 12, 278-287 (2012). doi: $10.1038 / \mathrm{nrc} 3236$

18. Sugiyama, A., Umetsu, M., Nakazawa, H., Niide, T., Onodera, T., Hosokawa, K., Hattori, S., Asano, R. \& Kumagai, K. A semi high-throughput method for screening small bispecific antibodies with high cytotoxicity. Sci. Rep. 7, 2862 (2017). doi: 10.1038/s41598-017-03101-4 
19. Fujii, H., Tanaka Y., Nakazawa, H., Sugiyama, A., Manabe, N., Shinoda, A., Shimizu, N., Hattori, T., Hosokawa, K., Sujino, T., Ito, T., Niide, T., Asano, R., Kumagai, I. \& Umetsu, M. Compact SeahorseShaped T Cell-Activating Antibody for Cancer Therapy, Adv. Therap.1, 1700031 (2018). doi.org/10.1002/adtp.201700031

20. Patel, S.G., Sayers, E.J., He, L., Narayan, R., Williams, T.L., Mills, E.M., Allemann, R.K., Luk, L.Y.P., Jones, A.T. \& Tsai, Y.H. Cell-penetrating peptide sequence and modification dependent uptake and subcellular distribution of green florescent protein in different cell lines. Sci. Rep. 9, 6298 (2019). doi: $10.1038 /$ s41598-019-42456-8

21. Mitchell, D.J., Kim, D.T., Steinman, L., Fathman, C.G. \& Rothbard, J.B. Polyarginine enters cells more efficiently than other polycationic homopolymers. J. Pept. Res. 56, 318-325 (2000). doi: 10.1034/j.1399-3011

22. Nakazawa, H., Kim, D.M., Matsuyama, T., Ishida, N., Ikeuchi, A., Ishigaki, Y., Kumagai, I. \& Umetsu, M. Hybrid Nanocellulosome Design from Cellulase Modules on Nanoparticles: Synthetic Effect of Catalytically Divergent Cellulase Modules on Cellulose Degradation Activity. ACS catalysis 3, 13421348 (2013). doi.org/10.1021/cs400012v

23. Siegmund, V., Schmelz, S., Dickgiesser, S., Beck, J., Ebenig, A., Fittler, H., Frauendorf, H., Piater, B., Betz, U.A., Avrutina, O., Scrima, A., Fuchsbauer, H.L. \& Kolmar, H. Locked by Design: A Conformationally Constrained Transglutaminase Tag Enables Efficient Site-Specific Conjugation. Angew. Chem. Int. Ed. Engl.54, 13420-13424 (2015). doi: 10.1002/anie.201504851.

24. Schumacher, D., Hackenberger, C.P., Leonhardt, H. \& Helma, J. Current Status: Site-Specific Antibody Drug Conjugates. J. Clin. Immunol.36, 100-107 (2016). doi: 10.1007/s10875-016-0265-6.

25. Strop, P. Versatility of microbial transglutaminase. Bioconjugate Chem. 25, 855-862 (2014). doi: 10.1021/bc500099v.

26. Takahara, M., Wakabayashi, R., Minamihata, K., Goto, M. \& Kamiya, N. Primary Amine-Clustered DNA Aptamer for DNA-Protein Conjugation Catalyzed by Microbial Transglutaminase. Bioconjugate Chem. 28, 2954-2961 (2017). doi: 10.1021/acs.bioconjchem.7b00594

27. Takahara, M., Wakabayashi, R., Minamihata, K., Goto, M. \& Kamiya, N. Design of Lipid-Protein Conjugates Using Amphiphilic Peptide Substrates of Microbial Transglutaminase. ACS Appl. Mater. Interfaces 1, 1823-1829 (2018). doi.org/10.1021/acsabm.8b00271

28. Roovers, R.C., Laeremans, T., Huang, L., De Taeye, S., Verkleij, A.J., Revets, H., de Haard, H. J. \& van Bergen en Henegouwen, P. M. Efficient inhibition of EGFR signaling and of tumour growth by antagonistic anti-EFGR Nanobodies. Cancer Immunol. Immunother. 56, 303-317 (2007). doi: 10.1007/s00262-006-0180-4

29. Nakazawa, H., Okada, K., Kobayashi, R., Kubota, T., Onodera, T., Ochiai, N., Omata, N., Ogasawara, W., Okada, $\mathrm{H}$. and Morikawa, Y. Characterization of the catalytic domains of Trichoderma reesei endoglucanase I, II, and III, expressed in Escherichia coli. Appl. Microbiol. Biotechnol.81, 681-689 (2008). doi: 10.1007/s00253-008-1667-z 
30. Ward, E.S. Antibody Engineering Using Escherichia coli as Host. Adv. Pharmacol.24, 1-20 (1993). doi: 10.1016/s1054-3589(08)60931-x

31. Wang, Y.H., Hou, Y.W. \& Lee, H.J. An intracellular delivery method for siRNA by an arginine-rich peptide. J. Biochem. Biophys. Methods 70, 579-586 (2007). doi: 10.1016/j.jbbm.2007.01.010

32. Lam, P. \& Steinmetz, N.F. Delivery of siRNA therapeutics using cowpea chlorotic mottle virus-like particles. Biomater. Sci. 7, 3138-3142 (2019). doi: 10.1039/c9bm00785g.

33. Opaliński, Ł., Szymczyk, J., Szczepara, M., Kucińska, M., Krowarsch, D., Zakrzewska M. \& Otlewski, J. High Affinity Promotes Internalization of Engineered Antibodies Targeting FGFR1. Int. J. Mol. Sci. 19, 1435 (2018). doi: 10.3390/ijms19051435.

34. Nakase, I., Takeuchi, T., Tanaka, G. \& Futaki, S. Methodological and cellular aspects that govern the internalization mechanisms of arginine-rich cell-penetrating peptides. Adv. Drug. Deliv. Rev.60, 598607 (2008). doi: 10.1016/j.addr.2007.10.006.

35. Lu, Y., Wang, Y., Zhang, M., Liu, L., Li F., Zhang, J., Ye, M., Zhao, H., Zhao J., Yan, B., Yang, A., Zhang, R., Li, X. \& Ren, X. HER2-siRNA Delivered by EGFR-specific Single Chain Antibody Inhibits NSCLC Cell Proliferation and Tumor Growth. Oncotarget. 26, 23594-23607 (2016). doi: 10.18632/oncotarget.8053.

36. Boussif, O., Lezoualc'h, F., Zanta, M.A., Mergny, M.D., Scherman, D., Demeneix, B. \& Behr, J.P. A versatile vector for gene and oligonucleotide transfer into cells in culture and in vivo: polyethylenimine. Proc. Natl. Acad. Sci. U.S.A. 92, 7297-7301 (1995). doi: 10.1073/pnas.92.16.7297

\section{Figures}




\section{Figure 1}
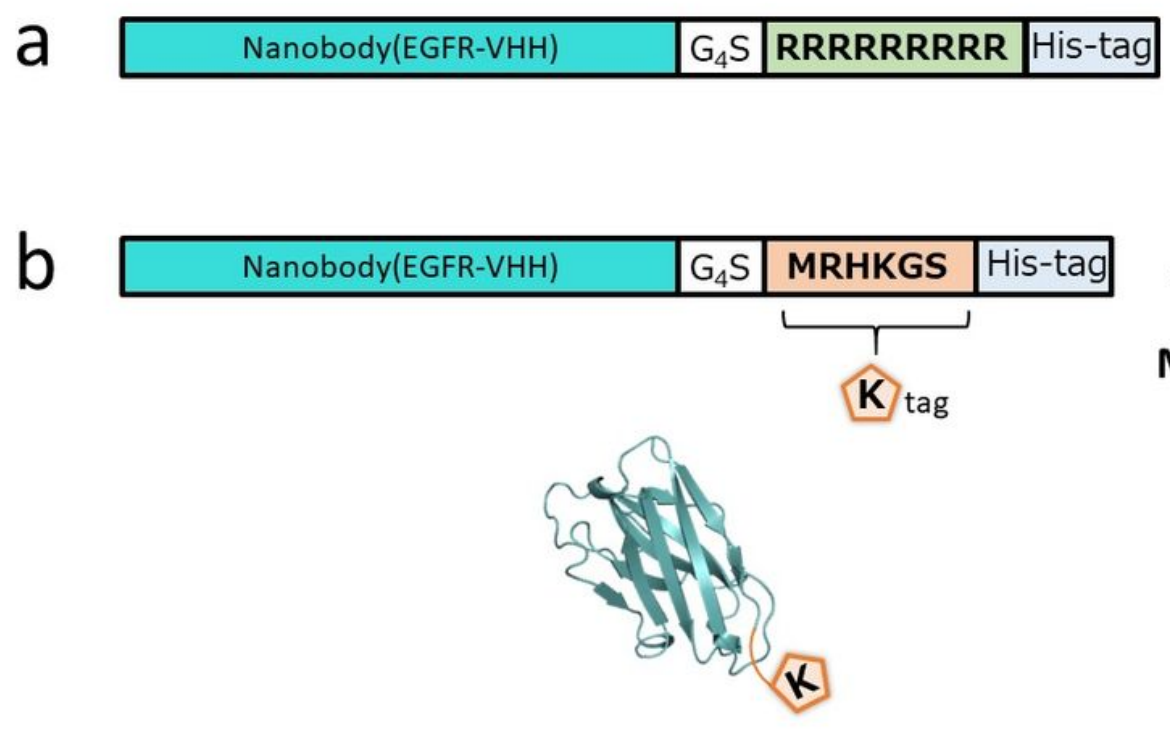

Nanobody-K

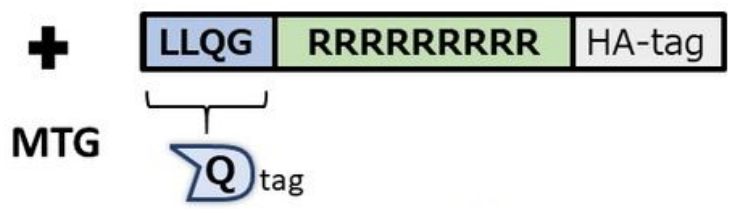

\section{Figure 1}

One-dimensional structure of the protein designed in this study. (a) Nanobody-R9 (b) Nanobody-K and QR9-HA. Orange color: la1 VHH (anti EGFR Nanobody), White color: G4S linker, Green color: R9 peptide, beige color: K-tag, light blue color: His-tag, Purple color: Q-tag, and light green color: HA-tag. 

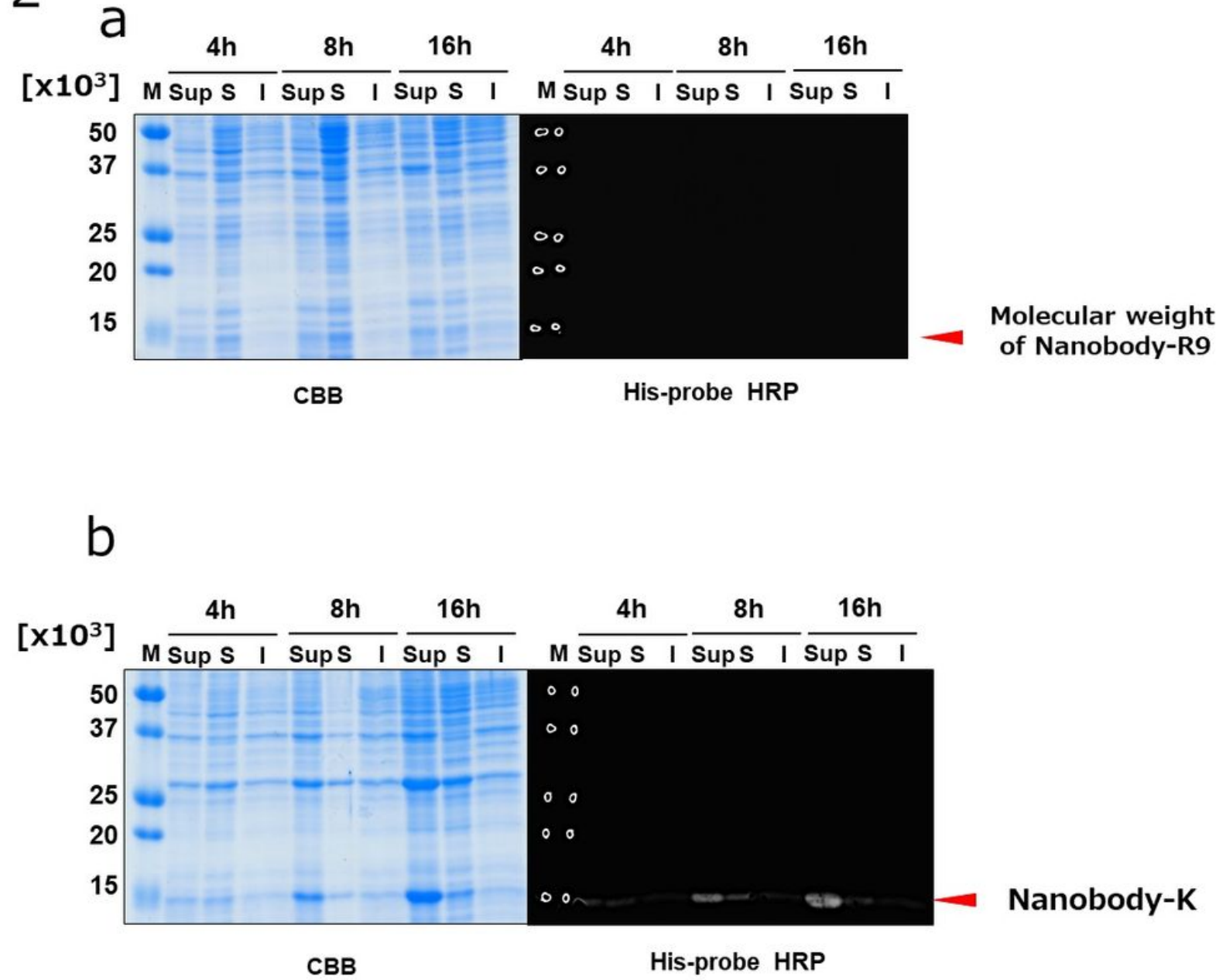

\section{Figure 2}

Expression analysis of Nanobody-R9 (a) and Nanobody-K (b) in E. coli. The figures show SDS-PAGE analysis (Right panels) using Coomassie Brilliant Blue (CBB) detection and western blot analysis (Right panels) using His-probe HRP detection of culture supernatant (Sup), intracellular soluble fraction (S), and intracellular insoluble fraction (I) under the induction at $28^{\circ} \mathrm{C}$ for $4-16 \mathrm{~h}$. M show Low molecular weight marker. The arrows indicate the deduced molecular weights of the target proteins. Complete gels and western blots are presented in Supplementary Figure 1. 


\section{Figure 3}

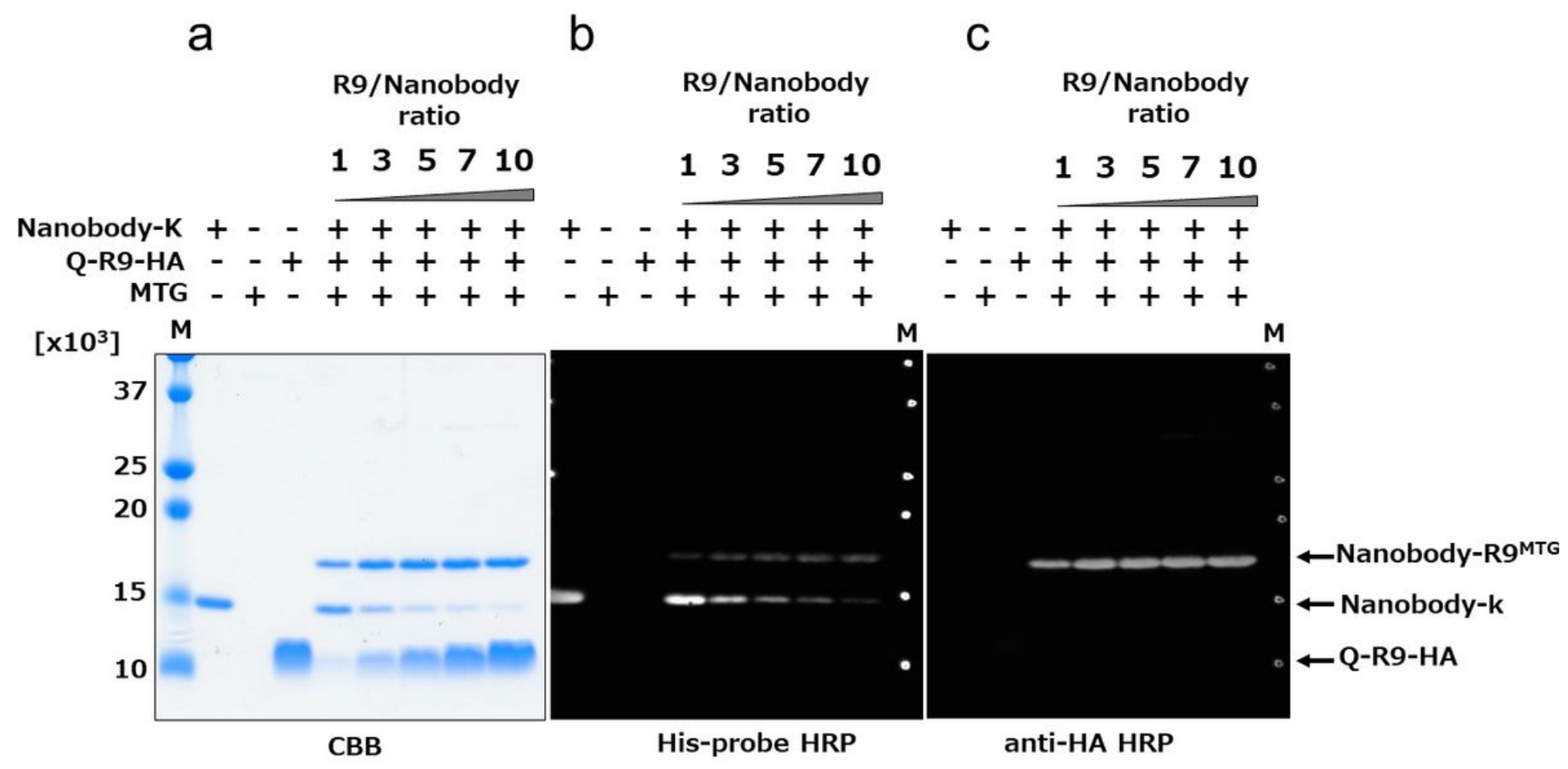

\section{Figure 3}

Enzymatic ligation of Nanobody and R9 by MTG. (a) SDS-PAGE by CBB staining; (b) western blot analysis using His-probe HRP; and (c) western blot analysis using anti-HA antibody. A total of 5-35 $\mu \mathrm{M}$ Q-R9-HA (1-10 times Nanobody concentration) was mixed with 5 mM Nanobody-K and 0.03 U mL-1 MTG and incubated at $20^{\circ} \mathrm{C}$ for $6 \mathrm{~h}$. The arrows indicate the deduced molecular weight of each protein. M indicate Low molecular weight marker for protein. 
Figure 4

a

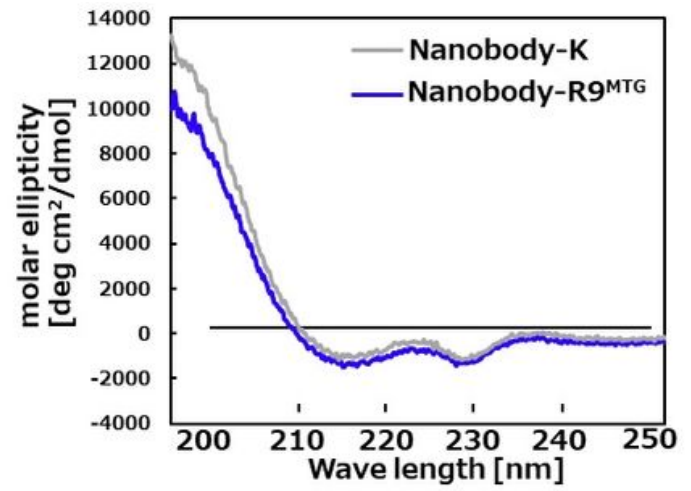

b

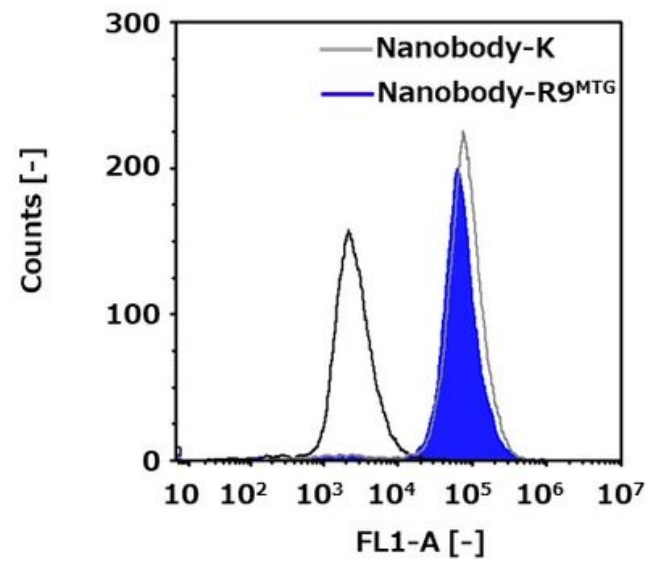

C

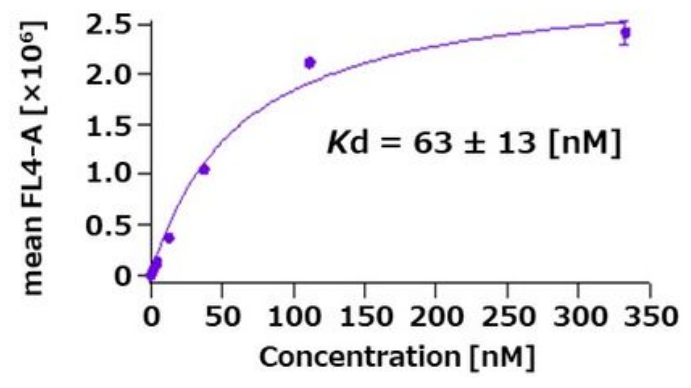

Figure 4

Physical properties of Nanobody-R9. (a) Analysis of Nanobody-R9MTG and Nanobody-K secondary structure by CD spectra. (b) Analysis of binding affinity of Nanobody-R9MTG and Nanobody-K by flow cytometry. (c) Calculation of Kd value of Nanobody-R9MTG by flow cytometry. 
Figure 5

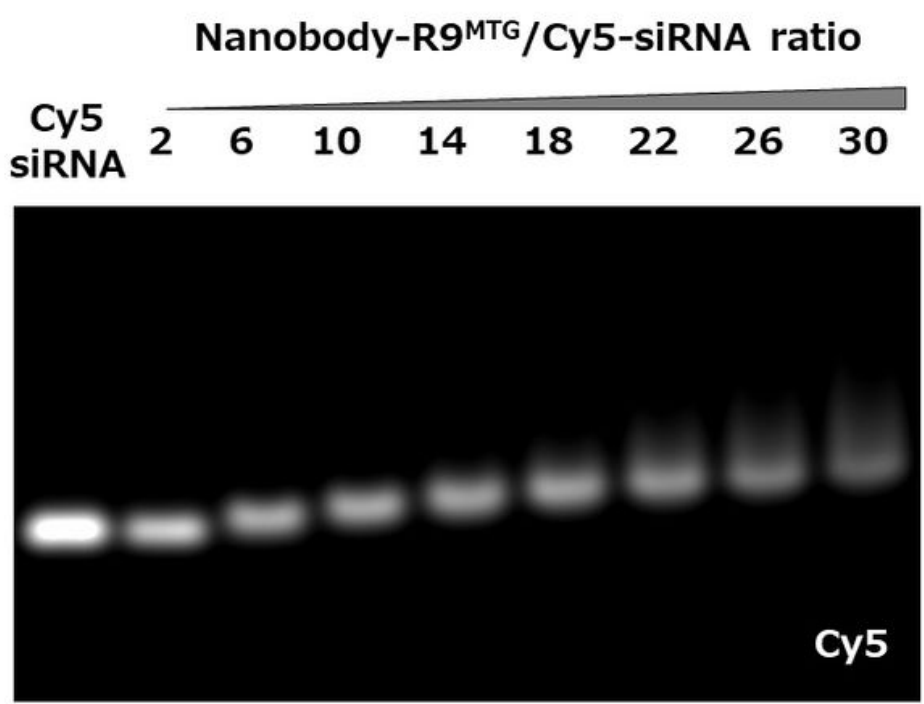

Figure 5

Interaction between siRNA and Nanobody-R9MTG. Electrophoretic mobility shift assay of NanobodyR9MTG and siRNA complex detected by Cy5. 


\section{Figure 6}

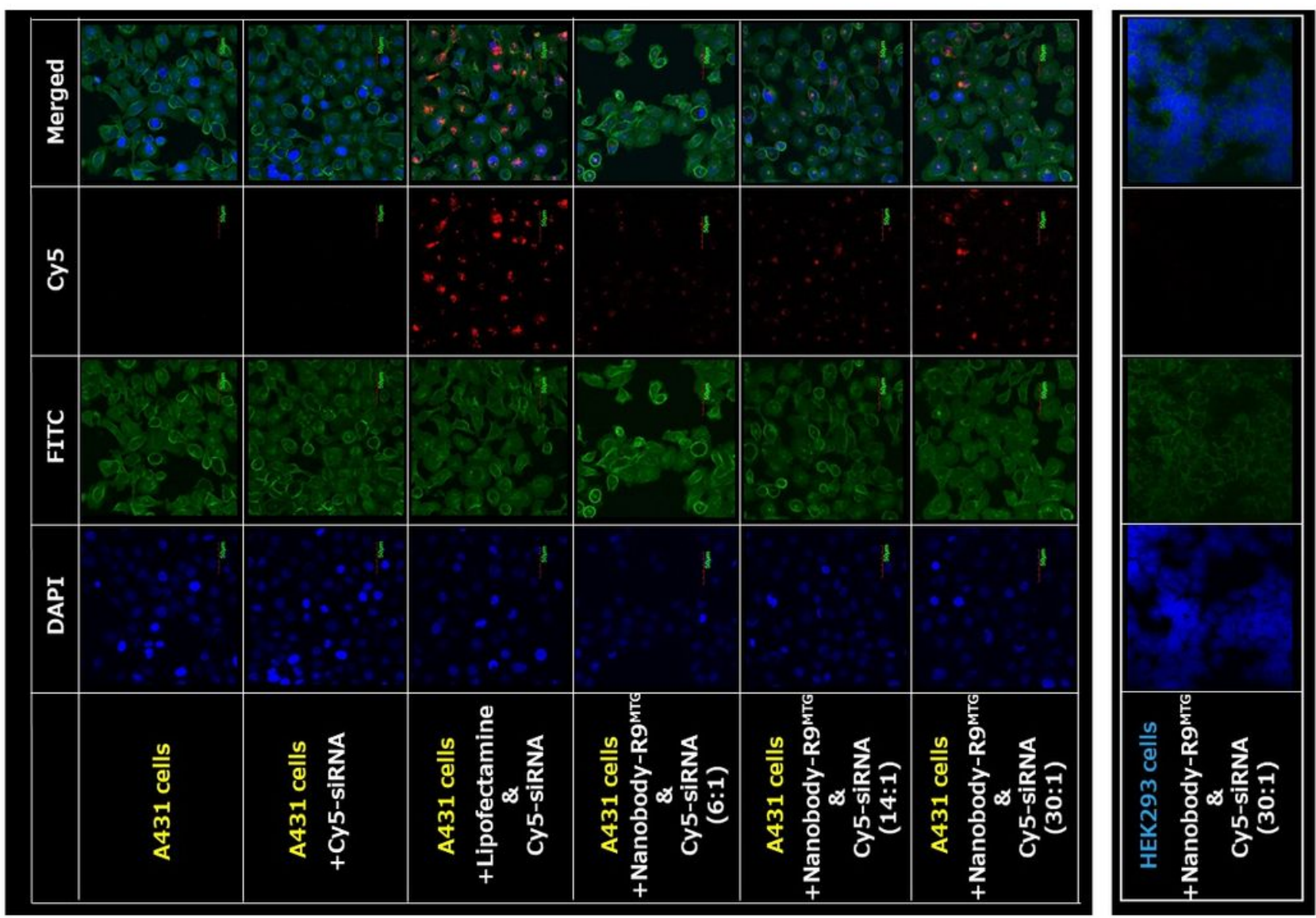

\section{Figure 6}

Delivery of siRNA by Nanobody-R9MTG into A431 cells. EGFR positive cells (A431) were mixed with Nanobody-R9MTG-Cy5 siRNA. Cell nuclei and membranes were stained with DAPI and Cell Mask ${ }^{\mathrm{TM}}$ Green Plasma Membrane stain. The fluorescence of the cells after the reaction was observed using a confocal microscope. HEK293 cells were used as EGFR negative cell. Lipofectamine is used as positive control. 


\section{Figure7}

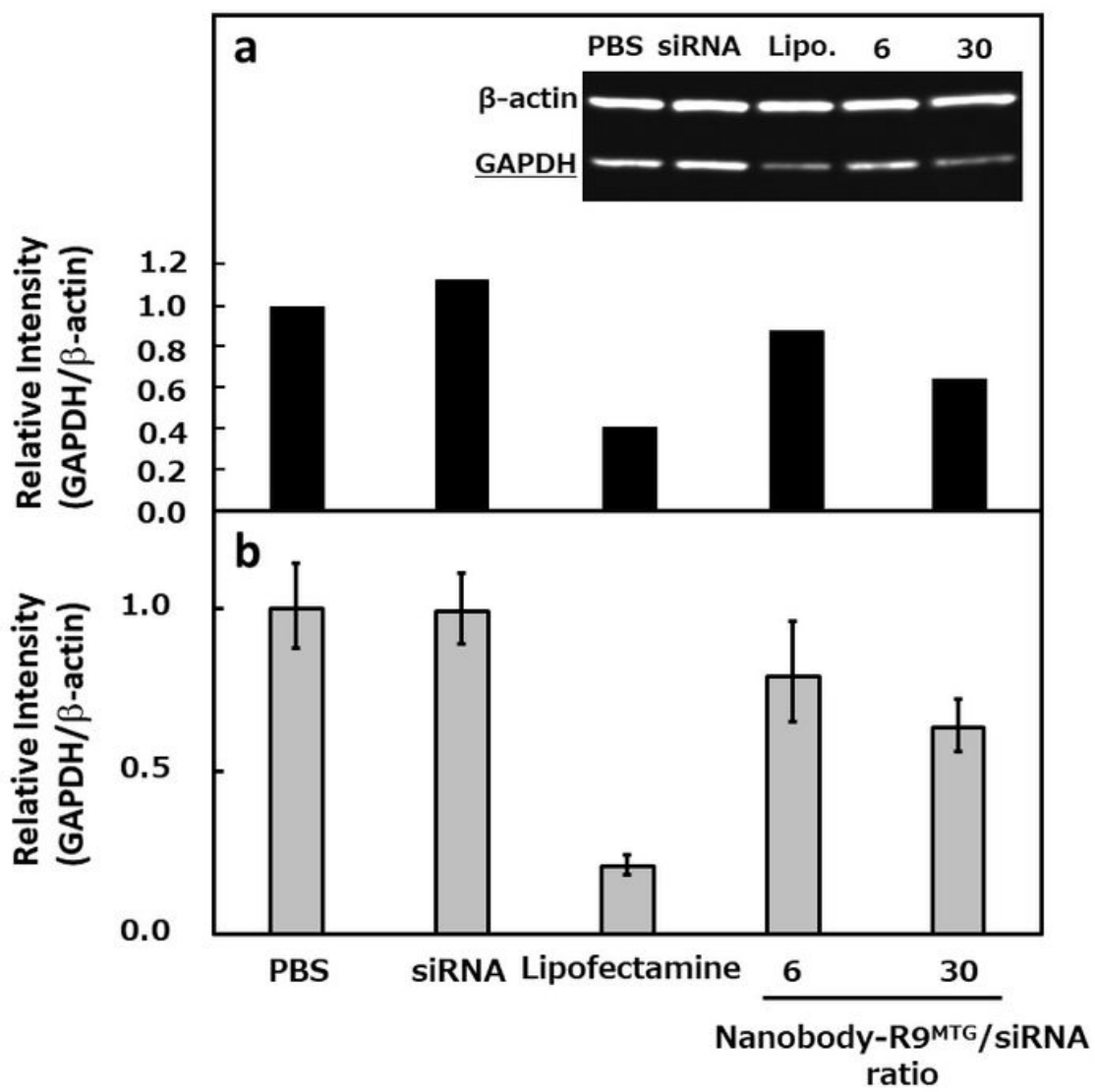

Figure 7

RNAi by Nanobody-R9MTG-siRNA (a) Western blot analysis of GAPDH in the A431 cell extract mixed with Nanobody-R9MTG-siRNA using anti-GAPDH monoclonal antibody. (b) Real time PCR of the transcript in A431 cell extract mixed with Nanobody-R9MTG-siRNA. Lipofectamine was used as a positive control. PBS, phosphate buffered saline, Lipo: lipofectamine, 6 and 30 indicate the NanobodyR9MTG/siRNA ratio.

\section{Supplementary Files}

This is a list of supplementary files associated with this preprint. Click to download.

- SupportingInformation.docx

- SIFigure2.tif

- SIFigure3.tif

- SIFigure4.tif 
- SIFigure1.tif

Page 22/22 